Bull. Korean Math. Soc. 48 (2011), No. 6, pp. 1119-1124

http://dx.doi.org/10.4134/BKMS.2011.48.6.1119

\title{
CHAOTIC PROPERTY OF WEIGHTED COMPOSITION OPERATORS
}

\author{
HAMid ReZAei
}

\begin{abstract}
In the present paper, we study the chaotic property of weighted composition operators acting on the holomorphic function space $H(\mathbb{U})$.
\end{abstract}

\section{Introduction}

A continuous linear operator $T$ acting on a separable Frechet space $X$ is called hypercyclic provided there exists a vector $x \in X$ such that its orbit $\operatorname{orb}(T, x)=\left\{T^{n} x: n=0,1, \ldots\right\}$ is dense in $X$. A periodic point for $T$ is a vector $x \in X$ such that $T^{n} x=x$ for some $n \in \mathbb{N}$. Finally, $T$ is said to be chaotic if it is hypercyclic and its set of periodic points is dense in $X$.

In 1929, Birkhoff [1] showed that the translation operator $T_{a}: H(\mathbb{C}) \rightarrow H(\mathbb{C})$ defined by $\left(T_{a} f\right)(z)=f(z+a), a \neq 0$, is hypercyclic on the Frechet space $H(\mathbb{C})$ of entire functions. This result was generalized by Godefroy and Shapiro [7] who proved that each operator on $H\left(\mathbb{C}^{N}\right)$ which commutes with all translations and is not a scalar multiple of the identity, is chaotic. Other classical examples of hypercyclic and chaotic operators are weighted shifts on $\ell^{p}$ spaces $[8,10]$ and adjoints of multiplication operators on Hilbert spaces of holomorphic functions $[7]$.

Let $\mathbb{U}$ stand for the open unit disk in $\mathbb{C}$. Each $\varphi \in H(\mathbb{U})$ and each holomorphic self-map $\psi$ of $\mathbb{U}$ induce a linear weighted composition operator $C_{\varphi, \psi}$ : $H(\mathbb{U}) \rightarrow H(\mathbb{U})$ defined by $\left(C_{\varphi, \psi} f\right)(z)=\varphi(z) f(\psi(z))$ with $f \in H(\mathbb{U})$ and $z \in \mathbb{U}$. In fact, we have $C_{\varphi, \psi}=M_{\varphi} C_{\psi}$, where $M_{\varphi}$ denotes the operator of multiplication by $\varphi$, and $C_{\psi}$ stands for the composition operator defined by $C_{\psi} f=f \circ \psi$ with $f \in H(\mathbb{U})$. Hence, the class of weighted composition operators includes the two important classes of composition operators and multiplication operators.

The chaotic property of composition operators on general holomorphic function spaces was studied in [12]. It is known that if $\psi$ is a holomorphic self-map

Received April 13, 2009; Revised October 3, 2009

2010 Mathematics Subject Classification. Primary 47B33; Secondary 47B38.

Key words and phrases. weighted composition operator, hypercyclic operator, DenjoyWolff point, Julia-Caratheodory theorem, linear-fractional model theorem, chaotic operator. 
of $\mathbb{U}$ and $C_{\psi}$ is hypercyclic on $H(\mathbb{U})$, then $\psi$ must be univalent and cannot have a fixed point in $\mathbb{U}$. Conversely, if $\psi$ is a univalent holomorphic self-map of $\mathbb{U}$ with no fixed point in $\mathbb{U}$, then $C_{\psi}$ is chaotic on $H(\mathbb{U})$ (see $[12$, Theorem 4.7]).

In this paper, we first extend the property of chaoticity to any nonzero multiple of $C_{\psi}$. Then we show that, under certain assumptions, $C_{\varphi, \psi}$ has a nonzero eigenvalue. This implies that the chaoticity of $C_{\varphi, \psi}$ can be derived from the chaoticity of $C_{\psi}$.

\section{Main result}

Let $\mathbb{P}$ stand for the open right half-plane in $\mathbb{C}$. In what follows, we will assume that $\psi$ is a holomorphic self-map of $\mathbb{U}$ and $\varphi$ is a holomorphic map on $\mathbb{U}$. Yousefi and the author [13, Proposition 2.1] showed that if the weighted composition operator $C_{\varphi, \psi}$ is hypercyclic, then $\psi$ is univalent and has no fixed point in $\mathbb{U}$. Moreover, the function $\varphi$ must be nonzero on all of $\mathbb{U}$. Since we aim to study chaotic weighted composition operators, we will focus on univalent holomorphic self-maps $\psi$ of $\mathbb{U}$ with no fixed point in $\mathbb{U}$, and we will assume that $\varphi$ is nonzero on all of $\mathbb{U}$.

In this case, the Denjoy-Wolff Theorem $[2,11,12]$ implies that there is a unique point $w \in \partial \mathbb{U}$ (called the Denjoy-Wolff point) such that $\psi_{n} \rightarrow w$ uniformly on compact subsets of $\mathbb{U}$ where $\psi_{n}=\psi \circ \psi \circ \cdots \circ \psi, n$ times. Moreover, the angular derivative $\psi^{\prime}(w)$ of $\psi$ at $w$ is real, positive, and less than or equal to one.

The mapping $\psi$ is called hyperbolic if $0<\psi^{\prime}(w)<1$ and parabolic if $\psi^{\prime}(w)=$ 1. Parabolic maps can be further distinguished into parabolic-automorphic and parabolic-nonautomorphic type depending on whether the distance in the hyperholic metric between successive points $\psi_{n}(z)$ stays bounded away from zero or not.

The Linear Fractional Model Theorem $([2,11,12,4])$. Let $\psi$ be a univalent, holomorphic self-map of $\mathbb{U}$ which has no fixed point in $\mathbb{U}$. Then there exist a univalent map $\sigma: \mathbb{U} \rightarrow \Omega(\Omega$ being either $\mathbb{P}$ or $\mathbb{C})$ and a linear-fractional map $\hat{\psi}$ mapping $\Omega$ onto $\Omega$ such that $\sigma \circ \psi=\hat{\psi} \circ \sigma$. In particular, there are the following possibilities:

(a) If $\psi$ is hyperbolic, then $\hat{\psi}$ can be taken to be a dilation, $\hat{\psi}(z)=r z$ for some $0<r<1$, and $\sigma(\mathbb{U}) \subseteq \Omega=\mathbb{P}$.

(b) If $\psi$ is parabolic-automorphic, then $\hat{\psi}$ can be taken to be a translation, $\hat{\psi}(z)=z \pm i$, and $\sigma(\mathbb{U}) \subseteq \Omega=\mathbb{P}$.

(c) If $\psi$ is parabolic-nonautomorphic, then $\hat{\psi}$ can be taken to be a translation, $\hat{\psi}(z)=z+1$, and $\sigma(\mathbb{U}) \subseteq \Omega=\mathbb{C}$.

Moreover, $\sigma(\mathbb{U})$ is a so-called fundamental set for $\Omega$ [5], i.e., for each compact subset $K$ of $\Omega$ there exists an $n$ such that $\hat{\psi}_{n}(K) \subseteq \sigma(\mathbb{U})$. 
Note that this theorem asserts that in some sense linear fractional maps are models for univalent self-maps of $\mathbb{U}$ that fix no point of $\mathbb{U}$.

Assume that $T$ and $S$ are two continuous linear operators on separable Frechet spaces $X$ and $Y$. We will call $S$ a quasi-factor of $T$, and $T$ a quasiextension of $S$ if there exists a continuous linear operator $V: X \rightarrow Y$ which has a dense range and satisfies $V T=S V$. It is easy to check that if $T$ is chaotic on $X$, then $S$ is also chaotic on $Y$.

The following lemma will be crucial to our result on the chaotic property of weighted composition operators. Therein, the operator $C_{\psi}$ acts on $H(\mathbb{U})$ and the operator $T_{a}$ acts on $H(\mathbb{C})$.

Lemma 2.1. Let $\psi$ be a univalent holomorphic self-map of $\mathbb{U}$ with no fixed points in $\mathbb{U}$. Then the operator $C_{\psi}$ is a quasi-factor of the translation $T_{a}$ for some complex number $a \neq 0$.

This result was already used by Shapiro in [12], but it is not stated explicitly there. For this reason, we sketch a proof of this lemma:

Proof. By the Linear Fractional Model Theorem, there exist a linear-fractional map $\hat{\psi}$ and a univalent map $\sigma: \mathbb{U} \rightarrow \mathbb{C}$ such that $\sigma \circ \psi=\hat{\psi} \circ \sigma$. We have two possibilities (corresponding to the hyperbolic and parabolic case):

Case I: $\hat{\psi}(z)=r z$ for some $0<r<1$, and $\sigma(\mathbb{U}) \subseteq \mathbb{P}$. Consider the following chain of maps:

$$
\mathbb{U} \stackrel{\sigma}{\longrightarrow} \mathbb{P} \stackrel{\hat{\psi}}{\longrightarrow} \mathbb{P} \stackrel{L}{\longrightarrow} \mathbb{S} \stackrel{j}{\longrightarrow} \mathbb{C}
$$

where $L=\log$ is the principal branch of logarithm taking $\mathbb{P}$ univalently onto the horizontal strip $\mathbb{S}=\left\{w \in \mathbb{C}:|\operatorname{Im}(w)|<\frac{\pi}{2}\right\}$, and $j$ is the embedding map from $\mathbb{S}$ to $\mathbb{C}$. Let $a=\log r, f=j \circ L \circ \sigma, \tau_{a}(z)=z+a$, and $V=C_{f}$. Then $f \circ \psi=j \circ L \circ \sigma \circ \psi=j \circ L \circ \hat{\psi} \circ \sigma=\tau_{a} \circ j \circ L \circ \sigma=\tau_{a} \circ f$. Hence the following diagram is commutative:

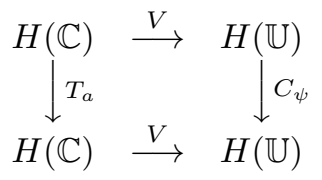

that is $V T_{a}=C_{\psi} V$. The set $\mathbb{G}=f(\mathbb{U})$ is a simply-connected domain. As a consequence of Runge's theorem the polynomials are dense in $H(\mathbb{G})$. This implies that $V$ has a dense range, and thus $C_{\psi}$ is a quasi-factor of $T_{a}$.

Case II: $\hat{\psi}(z)=z+a$ for some $a \in \mathbb{C}$. Consider the following chain of maps:

$$
\mathbb{U} \stackrel{\sigma}{\longrightarrow} \mathbb{C} \stackrel{\tau_{a}=\hat{\psi}}{\longrightarrow} \mathbb{C}
$$

where $\tau_{a}(z)=z+a$ as before. Let $f=\sigma$ and $V=C_{f}$. Then $f \circ \psi=\sigma \circ \psi=$ $\hat{\psi} \circ \sigma=\tau_{a} \circ f$, and the preceding diagram is again commutative. As in the last case, $V$ has a dense range, and its follows that $C_{\psi}$ is a quasi-factor of $T_{a}$. 
Theorem 2.2. Suppose $\psi$ is a univalent self-map of $\mathbb{U}$ and $\lambda$ is a nonzero scalar. Then $\lambda C_{\psi}$ is chaotic if and only if $\psi$ has no fixed point in $\mathbb{U}$.

Proof. If $\psi$ has a fixed point $z \in \mathbb{U}$, then it is easy to check that the linear functional $\Lambda_{z}: H(\mathbb{U}) \longrightarrow \mathbb{C}$ by $\Lambda_{z}(f)=f(z)$ is an eigenvector of $\lambda C_{\psi}^{*}$, and hence $\lambda C_{\psi}$ is not hypercyclic [7]. Now assume that $\psi$ has no fixed point in $\mathbb{U}$. Then by the last lemma, $C_{\psi}$ is a quasi-factor to a translation $T_{a}$ for some complex number $a \neq 0$ and consequently $\lambda C_{\psi}$ is also a quasi-factor of $\lambda T_{a}$. But by [7, Theorem 6.2], $\lambda T_{a}$ is chaotic, and hence $\lambda C_{\psi}$ is also chaotic.

Definition 2.3. For any $w \in \overline{\mathbb{U}}$ and positive real number $\beta$, we denote by $\operatorname{Lip}_{\beta}(w)$, the class of holomorphic functions $\varphi$ satisfying

$$
|\varphi(z)-\varphi(w)|=O\left(|z-w|^{\beta}\right) \quad(z \rightarrow w) .
$$

For example, if $\varphi \in H(\mathbb{U})$ is analytic at $w$, then $\varphi \in \operatorname{Lip}_{\beta}(w)$ for $\beta \in(0,1]$. Moreover, if $\varphi^{(i)}(w)$ exists and equals to zero for $i=1, \ldots, n$, then $\varphi \in \operatorname{Lip}_{\beta}(w)$ for $\beta \in(0, n+1]$.

Yousefi and the author [13, Proposition 2.4] have shown that if $\varphi \in \operatorname{Lip}_{\beta}(w)$ for some real number $\beta>0, \varphi(z) \neq 0$ for all $z \in \mathbb{U}$, and $\varphi(w) \neq 0$, where $w \in \partial \mathbb{U}$ is the Denjoy-Wolff fixed point of the univalent self map $\psi$, then $g(z)=\prod_{n=0}^{\infty} \frac{1}{\varphi(w)} \varphi\left(\psi_{n}(z)\right)$ defines a non-vanishing holomorphic function on $\mathbb{U}$ and indeed an eigenfunction for $C_{\varphi, \psi}$ whenever $\psi$ is hyperbolic and $\beta>0$ or $\psi$ is a parabolic automorphism and $\beta>1$. Moreover $C_{\varphi, \psi}$ is hypercyclic if $|\varphi(w)|=1$.

We extend these results from [13] in two directions: we eliminate the requirement that $|\varphi(w)|=1$, and, in the parabolic case, we allow $\psi$ be any map of parabolic-automorphic type (but we do require $\beta=2$ ).

Lemma 2.4. The series $\sum_{n=1}^{+\infty}\left|\psi_{n}(z)-w\right|^{\beta}$ converges uniformly on compact subsets of $\mathbb{U}$, whenever $\psi$ is of hyperbolic type and $\beta>0$, or $\psi$ is of parabolicautomorphic type and $\beta=2$.

Proof. If $\psi$ is hyperbolic and $w \in \partial \mathbb{U}$, then $0<\psi^{\prime}(w)<1$, and by the JuliaCaratheodory inequality [2, Theorem 3] we have

$$
\frac{|\psi(z)-w|^{2}}{1-|\psi(z)|^{2}}<\psi^{\prime}(w) \frac{|z-w|^{2}}{1-|z|^{2}} \quad(z \in \mathbb{U}) .
$$

By substituting $\psi_{n}(z)$ for $z$, we obtain

$$
\frac{\left|\psi_{n}(z)-w\right|^{2}}{1-\left|\psi_{n}(z)\right|^{2}}<\left(\psi^{\prime}(w)\right)^{n} \frac{|z-w|^{2}}{1-|z|^{2}} \quad(z \in \mathbb{U}, n \geq 0) .
$$

Now if $K$ is a compact subset of $\mathbb{U}$, then the right hand side of the above inequality is bounded on $K$. Hence it follows that

$$
\left|\psi_{n}(z)-w\right|<\mathrm{const} \cdot\left(\psi^{\prime}(w)\right)^{\frac{n}{2}} \quad(z \in K) .
$$

Thus in this case, $\sum_{n=0}^{\infty}\left|\psi_{n}(z)-w\right|^{\beta}$ converges uniformly on $K$ for $\beta>0$. 
Next, let $\psi$ be of parabolic-automorphic type. The Linear Fractional Model Theorem $[2,11,12]$ then provides a function $\sigma$ holomorphic on $\mathbb{U}$ with values in the right half-plane such that $\sigma \circ \psi=\sigma+i b$ for some real $b \neq 0$. Hence $\sigma \circ \psi_{n}=\sigma+n i b$. Let $K$ be an arbitrary compact subset of $U$. For $n \geq 1$, pick $z_{n} \in K$ such that $\left|\psi_{n}\left(z_{n}\right)\right| \leq\left|\psi_{n}(z)\right|$ for all $z \in K$.

The Blaschke condition for a sequence in $\mathbb{U}$ is equivalent, via the map $w=$ $\frac{1+z}{1-z}$, to the condition

$$
\sum_{n} \frac{\operatorname{Re}\left(w_{n}\right)}{\left|1+w_{n}\right|^{2}}<\infty
$$

for sequences $\left(w_{n}\right)$ in $\mathbb{P}$. Since the sequence $\left(\sigma\left(z_{n}\right)\right)$ is bounded, $(3)$ is satisfied by the sequence $w_{n}=\sigma\left(z_{n}\right)+n i b$. Hence there exists a non-constant bounded holomorphic function $F$ on the right half-plane whose zeros are the numbers $w_{n}$ (see [5, Theorem 11.3]). Since $\sigma(\mathbb{U}) \subseteq \mathbb{P}$, the function $f=F \circ \sigma$ is then a non-constant bounded holomorphic function on $\mathbb{U}$, and for $n \geq 1$ :

$$
f\left(\psi_{n}\left(z_{n}\right)\right)=F\left(\sigma\left(\psi_{n}\left(z_{n}\right)\right)\right)=F\left(\sigma\left(z_{n}\right)+n i b\right)=0 .
$$

It follows that the sequence $\left(\psi_{n}\left(z_{n}\right)\right)$ satisfies the Blaschke condition on $\mathbb{U}$, that is

$$
\sum_{n=0}^{+\infty}\left(1-\left|\psi_{n}\left(z_{n}\right)\right|^{2}\right)<+\infty
$$

On the other hand, the Julia-Caratheodory inequality implies that

$$
\left|\psi_{n}(z)-w\right|^{2} \leq \mathrm{const} \cdot\left(1-\left|\psi_{n}(z)\right|^{2}\right) \leq \mathrm{const} \cdot\left(1-\left|\psi_{n}\left(z_{n}\right)\right|^{2}\right)
$$

on $K$. Thus in this case, $\sum_{n=0}^{\infty}\left|\psi_{n}(z)-w\right|^{2}$ converges uniformly on $K$ and the proof is complete.

Our main result is now the following.

Theorem 2.5. Let $\psi$ be a holomorphic self-map of $\mathbb{U}$ with Denjoy-Wolff fixed point $w \in \partial \mathbb{U}$. Suppose that $\varphi \in \operatorname{Lip}_{\beta}(w)$ is holomorphic and nonzero on all of $\mathbb{U}$, and that $\varphi(w) \neq 0$. Then $C_{\varphi, \psi}$ is chaotic, whenever $\psi$ is of hyperbolic type and $\beta>0$, or $\psi$ is of parabolic-automorphic type and $\beta=2$.

Proof. Assume that $\varphi \in \operatorname{Lip}_{\beta}(w)$ for some real number $\beta$, and let $K$ be a compact subset of $\mathbb{U}$. Since $\psi_{n} \rightarrow w$ uniformly on $K$, by substituting $\psi_{n}(z)$ for $z$ in $(1)$, we get

$$
\left|\varphi(w)-\varphi\left(\psi_{n}(z)\right)\right|=O\left(\left|w-\psi_{n}(z)\right|^{\beta}\right) \quad(z \in K, n \rightarrow \infty),
$$

whence

$$
\left|1-\frac{1}{\varphi(w)} \varphi\left(\psi_{n}(z)\right)\right|=O\left(\frac{1}{|\varphi(w)|}\left|w-\psi_{n}(z)\right|^{\beta}\right) \quad(z \in K, n \rightarrow \infty) .
$$


Now, if $\psi$ is hyperbolic and $\beta>0$ or $\psi$ is parabolic-automorphic and $\beta=2$, then Lemma 2.4 implies that $\sum_{n=0}^{\infty}\left|1-\frac{1}{\varphi(w)} \varphi\left(\psi_{n}(z)\right)\right|<\infty$, and consequently

$$
g(z)=\prod_{n=0}^{\infty} \frac{1}{\varphi(w)} \varphi\left(\psi_{n}(z)\right)
$$

converges uniformly on $K$. Thus $g$ defines a nonzero holomorphic function on $\mathbb{U}$ satisfying $\varphi(z) g(\psi(z))=\varphi(w) g(z)$. Hence $C_{\varphi, \psi} g=\varphi(w) g$, i.e., $g$ is an eigenfunction of $C_{\varphi, \psi}$ to the nonzero eigenvalue $\varphi(w)$.

Consequently, $C_{\varphi, \psi} M_{g}=\varphi(w) M_{g} C_{\psi}$. Note that the range of the multiplication operator $M_{g}$ is dense in $H(\mathbb{U})$ because the function $g(z)$ does not vanish on $\mathbb{U}$. Hence $C_{\varphi, \psi}$ is a quasi-factor of $\varphi(w) C_{\psi}$. Since $\varphi(w) C_{\psi}$ is chaotic, by Theorem 2.2, it follows that $C_{\varphi, \psi}$ is also chaotic.

Acknowledgments. The author is very grateful to the referee for many helpful suggestions and interesting comments about the paper.

\section{References}

[1] G. D. Birkhoff, Demonstration d'un theoreme elementaire sur les fonctions entieres, C. R. Acad. Sci. Paris 189 (1929), 473-475.

[2] P. S. Bourdon and J. H. Shapiro, Cyclic phenomena for composition operators, Mem. Amer. Math. Soc. 125 (1997), no. 596, x+105 pp.

[3] K. C. Chan and J. H. Shapiro, The cyclic behavior of translation operator on Hilbert spaces of entire functions, Indiana Univ. Math. J. 40 (1991), no. 4, 1421-1449.

[4] C. C. Cowen, Iteration and the solution of functional equations for functions analytic In the unit disk, Trans. Amer. Math. Soc. 265 (1981), no. 1, 69-95.

[5] P. L. Duren, Theory of $H^{p}$ Spaces, Pure and Applied Mathematics, Vol. 38, Academic Press, New York, 1970; reprinted by Dover, 2000.

[6] R. M. Gethner and J. H. Shapiro, Universal vectors for operators on spaces of holomorphic functions, Proc. Amer. Math. Soc. 100 (1987), no. 2, 281-288.

[7] G. Godefroy and J. H. Shapiro, Operators with dense invariant cyclic vector manifolds, J. Funct. Anal. 98 (1991), no. 2, 229-269.

[8] K.-G. Grosse-Erdmann, Hypercyclic and chaotic weighted shifts, Studia Math. 139 (2000), no. 1, 47-68.

[9] G. R. MacLane, Sequences of derivatives and normal families, J. Analyse Math. 2 (1952), 72-87.

[10] H. N. Salas, Hypercyclic weighted shifts, Trans. Amer. Math. Soc. 347 (1995), no. 3, 993-1004.

[11] J. H. Shapiro, Composition Operators and Classical Function Theory, Springer-Verlag, New York, 1993.

[12] _ Notes on dynamics of linear operator, http://www. math.msu.edu/shapiro, (2001).

[13] B. Yousefi and H. Rezaei, Hypercyclic property of weighted composition operators, Proc. Amer. Math. Soc. 135 (2007), no. 10, 3263-3271.

Department of Mathematics

College of SCIEnCES

YASOUJ UNIVERSITY

YASOUJ 75918-74831, IRAN

E-mail address: rezaei@mail.yu.ac.ir 